\section{Inner segment ellipsoid band length is a prognostic factor in retinitis pigmentosa associated with EYS mutations: 5-year observation of retinal structure}

M Miyata, K Ogino, N Gotoh, S Morooka, T Hasegawa, M Hata and N Yoshimura
Department of

Ophthalmology and Visual Sciences, Kyoto University Graduate School of Medicine, Kyoto, Japan

Correspondence:

M Miyata, Department of Ophthalmology and Visual Sciences, Kyoto University Graduate School of Medicine, Sakyo-ku, Shogoin Kawahara-cho 54, Kyoto 606-8507, Japan Tel: +81 757513248 ; Fax: +81 757520933. E-mail: miyatam@kuhp. kyoto-u.ac.jp

Received: 5 March 2016 Accepted in revised form: 11 July 2016 Published online: 26 August 2016

\begin{abstract}
Purpose To evaluate whether the length of the inner segment ellipsoid (ISe) band can be used as a prognostic factor for disease course in retinitis pigmentosa (RP) patients with EYS mutations by observation over a period of 5 years.

Methods Twelve RP patients with EYS mutations were studied. The horizontal and vertical ISe length of the right eye was manually measured at five time points annually, using spectral domain optical coherence tomography. A regression line through the five points from baseline to the final measurement was drawn and the ratio of the length (\%) at each point to the baseline length was calculated; the slope was defined as the rate of ISe shortening (\%/year). The correlation between the rate of ISe shortening and age, visual acuity, and mean deviation (MD) value were evaluated. The intraclass correlation coefficient (ICC) for the measurements was calculated.

Results The mean rate of ISe shortening was $-4.65 \pm 2.89 \%$ per year and the decline was statistically significant. The rate of shortening was significantly negatively correlated with the baseline length $(P=0.046, r=0.58)$, but not with the baseline age, visual acuity, and MD value. The ICC $(2,1)$ was 0.999 .

Conclusions ISe of all RP patients with EYS mutations shortened during the 5 years of annual observation. The measurement of the length of ISe is a simple and convenient method with high repeatability, and the length is a sensitive prognostic factor for the rate of ISe shortening in RP patients with EYS mutations. Eye (2016) 30, 1588-1592; doi:10.1038/eye.2016.196; published online 26 August 2016
\end{abstract}

\section{Introduction}

Retinitis pigmentosa (RP), a set of retinal diseases featuring degeneration of rod and cone photoreceptors, varies with regard to the onset of symptoms, inheritance mode, fundus appearance, and prognosis, ${ }^{1}$ possibly because of differences in causative gene mutations. ${ }^{2,3}$ Sixty RP causative gene mutations were found. ${ }^{4}$

EYS is an important and common cause of RP in the Japanese, Spanish, British, Chinese, Israelis, and Palestinians. ${ }^{5-9}$ Furthermore, a report has described that EYS-associated RP patients share a relatively uniform phenotype with near-normal central visual function up to their 20s. ${ }^{10}$ We have previously reported that severity of RP patients with EYS mutations was relatively moderate among RP patients with various mutations. ${ }^{11}$ Thus, we encounter RP patients with EYS mutations at relatively high frequency in daily clinical consultation except detection for causative gene mutations, and RP patients with EYS mutations have a representative feature in RP. It is significant to investigate RP patients with EYS mutations.

Although a change in the retinal structure of RP patients with the same genetic mutations over the medium term (2 years) has been reported, ${ }^{12,13}$ no study, to our knowledge, has investigated the changes over the longer term (5 years). Because the change of retinal structure is gradual, it is necessary to assess the change using a long-term follow-up data. Moreover, it is important to understand the change when explaining the disease course to patients in clinical practice. 
It has been reported that evaluation of changes in the inner segment ellipsoid (ISe) band is useful for the assessment of retinal health, which is correlated with visual function. ${ }^{14-17}$ A previous 2-year study found that ISe decreases year-by-year in RP patients. ${ }^{18}$

From these perspectives, we conducted this study to evaluate whether the change of length of ISe over a longterm period in RP patients with EYS mutations can be used as a prognostic factor in predicting the disease course.

\section{Subjects and methods}

This study was approved by the ethics committee of Kyoto University Graduate School of Medicine (Kyoto, Japan). All study protocols adhered to the tenets of the Declaration of Helsinki. The nature of the study, and the possible risks and benefits of participation were explained to all study candidates. All subjects choosing to participate provided written informed consent.

\section{Subjects}

We performed gene analyses for 329 Japanese RP patients who visited the Department of Ophthalmology and Visual Sciences, Kyoto University Graduate School of Medicine, Kyoto, Japan between January 2011 and December 2012, and agreed to provide peripheral blood samples. ${ }^{5}$ All patients underwent comprehensive ophthalmological examinations, including measurement of the best-corrected visual acuity (BCVA) using a decimal visual acuity chart (Landolt chart), indirect ophthalmoscopy, slit-lamp biomicroscopy, SD-OCT (Spectralis HRA+OCT, Heidelberg Engineering, Heidelberg, Germany); mean deviation (MD) value at baseline calculated using a Humphrey field analyzer (HFA; 10-2 SITA Standard Program; Carl Zeiss Meditec, Jena, Germany) and $30 \mathrm{~Hz}$ flicker electroretinography (ERG) were also performed. ERG results were recorded according to the International Society for Clinical Electrophysiology of Vision standard protocol recommended in 2008 using LS-C (Mayo Co., Nagoya, Japan) and Neuropack MEB-2204 systems (Nihon Kohden, Tokyo, Japan). All BCVA data were converted to logarithm of the minimal angle of resolution (logMAR) for statistical analyses. Retinal specialists diagnosed RP using comprehensive ophthalmological examinations. In all the patients, EYS mutations were detected by nextgeneration sequencing. Inclusion criteria were: available SD-OCT images obtained over a period of 5 years, at five time points or more, on different days. Patients for whom ISe could not be detected on OCT images including dotted lines and those who had undergone intraocular surgery during the study period were excluded. Two investigators ( $\mathrm{MM}$ and $\mathrm{TH}$ ) determined whether the images were assessable. Those who had undergone intraocular surgery during the study period were also excluded.

\section{ISe band analysis}

The length of ISe on horizontal and vertical OCT B-scan crossing the fovea of the right eye was measured over 5 years using SD-OCT images of $30 \times 30^{\circ}$ scans $(\sim 9 \times 9 \mathrm{~mm})$ at five time points. The length of a healthy eye is $\sim 9000 \mu \mathrm{m}$, because ISe is not cut within range of the SD-OCT images. Representative measurements were used for analysis by averaging horizontal and vertical measurements. Measurements were performed obtained in a random order using the built-in measurement scale provided in the SD-OCT software (Heidelberg Engineering; Figure 1). Information pertaining to the date of measurement was masked. If the OCT images were obtained at more than five time points, we selected five images obtained at almost equal time intervals. We plotted this data, with the baseline date up to the final measurement date on the $x$ axis and the percentage of the length of ISe relative to that at baseline (\%) on the $y$ axis.

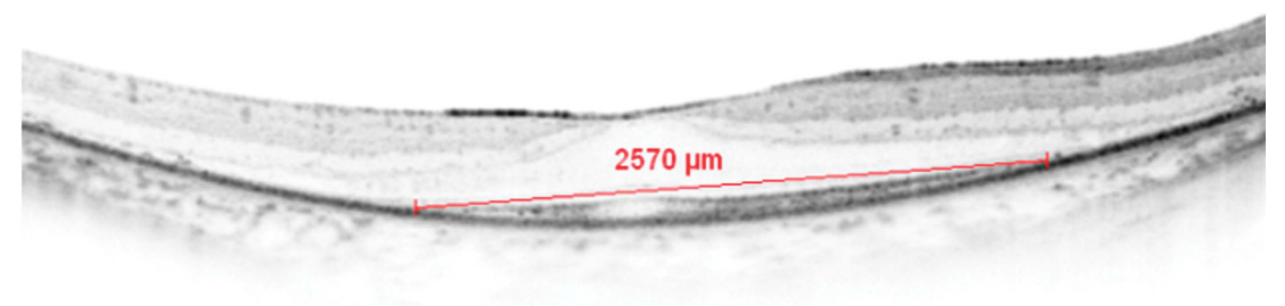

Figure 1 Spectral domain optical coherence tomography images. The length of the ISe band crossing the fovea was measured in a random order using the measurement scale provided in the spectral domain optical coherence software. Information pertaining to the date of measurement and type of gene was masked. 
A regression line through the five data points from baseline to the final measurement date was then drawn; the slope was defined as the rate of ISe shortening (\%/day). To improve comprehensibility, we converted the rate of ISe shortening (\%/day) to the rate of ISe shortening (\%/year) by multiplying by 365 . To assess the repeatability of measurements, another investigator measured the length of ISe of five patients selected at random in the same manner.

\section{Statistical analysis}

Data are presented as mean \pm SD where applicable. All statistical analyses were performed using SPSS version 21 (IBM, New York, NJ, USA). Student's $t$-tests were used to compare different data sets. Correlations were analyzed using Pearson's correlation coefficients. Linear regression analysis was performed to calculate the rate of ISe shortening. The intraclass correlation coefficient (ICC) value for the length of ISe measurements recorded by the two investigators (MM and $\mathrm{TH}$ ) was calculated to determine the reliability of measurements. A $P$-value of $<0.05$ was considered statistically significant.

\section{Results}

In total, 19 patients met the inclusion criteria for the study. Among these, ISe could not be detected in seven. Eventually, 12 patients were included in our analysis. In one patient, MD value at baseline was not available. Table 1 shows the patient characteristics. The observation period was $5.93 \pm 0.74$ years. The $\operatorname{ICC}(2,1)$ value for the length of ISe measurements was 0.999.

Figure 2 shows the relationship between the length of ISe at each time point relative to that at baseline and the followup duration. The rate of ISe shortening was $-4.65 \pm 2.89 \%$ per year. There was a significant difference between the derived rate of ISe shortening value and $0(P<0.001)$, indicating a significant decrease in the length of ISe. When the cases were analyzed individually, there were significant differences between the rate of ISe shortening and 0 in all patients. When we separated the horizontal and vertical measurements to analyze, the rate of horizontal and vertical ISe shortening was $-5.17 \pm 3.10 \%$ and $-4.26 \pm 2.98 \%$ per year, respectively. There were no differences in the rate between them $(P=0.70)$.

The rate of ISe shortening and the length of ISe at baseline were significantly correlated $(P=0.046, r=0.58)$, that is, the rate of ISe shortening value was high in patients with a small length of ISe value at baseline. However, there was no significant correlation between the rate of ISe shortening and age, logMAR visual acuity, and MD value at baseline $(P=0.84,0.30$, and 0.25 , respectively).
Table 1 Baseline characteristics of retinitis pigmentosa patients with EYS mutations included in this study

\begin{tabular}{|c|c|}
\hline Characteristics & Values \\
\hline Number of patients & 12 \\
\hline \multicolumn{2}{|l|}{ Patient age (years) } \\
\hline Mean $\pm S D$ & $45.6 \pm 8.6$ \\
\hline Range & $34-63$ \\
\hline \multicolumn{2}{|l|}{ Patient sex, no. (\%) } \\
\hline Male & $3(25)$ \\
\hline Female & $9(75)$ \\
\hline \multicolumn{2}{|c|}{ Visual acuity in the right eye, $\log M A R$} \\
\hline Mean \pm SD & $0.040 \pm 0.143$ \\
\hline Range & $-0.176-0.222$ \\
\hline \multicolumn{2}{|c|}{ Axial length in the right eye $(\mathrm{mm})$} \\
\hline Mean \pm SD & $24.51 \pm 0.99$ \\
\hline Range & 22.7-25.9 \\
\hline \multicolumn{2}{|c|}{$\begin{array}{l}\text { Mean deviation value as per Humphrey 10-2 visual field analysis ( } d B \text {; } \\
n=11 \text { ) }\end{array}$} \\
\hline Mean \pm SD & $-15.12 \pm 7.16$ \\
\hline Range & -25.36 to -1.11 \\
\hline \multicolumn{2}{|c|}{ The length of the inner segment ellipsoid band measurement ( $\mu \mathrm{m}$} \\
\hline Mean \pm SD & $3137 \pm 2350$ \\
\hline Range & 481-7589 \\
\hline Pseudophakia, no. (\%) & $1(8)$ \\
\hline
\end{tabular}

Abbreviations: logMAR, logarithm of the minimal angle of resolution; $\mathrm{SD}$, standard deviation.

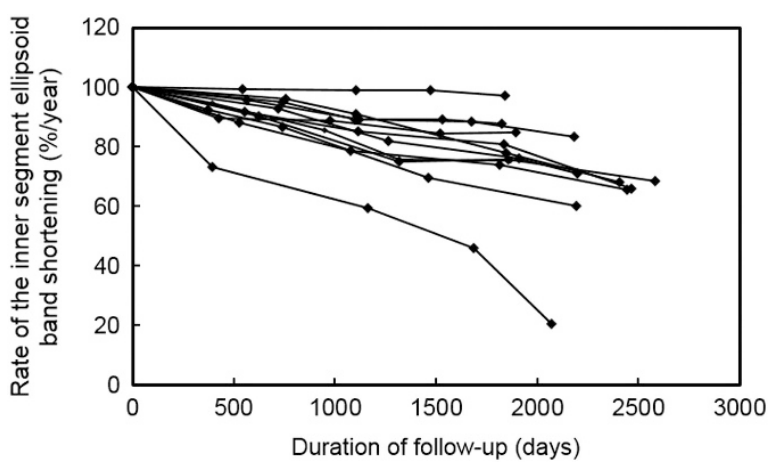

Figure 2 Relationship between the percentage length of the ISe band at the observation point relative to that at baseline and the follow-up duration. The mean \pm SD rate of the ISe band shortening is $-4.65 \pm 2.89 \%$ per year.

The decrease in the MD value varied; the mean decrease was $3.09 \pm 3.62 \mathrm{~dB}$ in the same 5 -year period. Among 12 patients, six decreased by $>2 \mathrm{~dB}$ and six decreased by $<2 \mathrm{~dB}$. The rate of ISe shortening was $-5.17 \pm 4.97 \%$ and $-3.52 \pm 1.17 \%$ per year, respectively. There were no significant differences of the rate between the two groups $(P=0.56)$. 


\section{Discussion}

This study evaluated whether the length of ISe, which is correlated with visual function, changes over a long-term period in RP patients with EYS mutations for the first time. The length of ISe of the most RP patients with EYS mutations shortened during the 5 years of annual observations and the rate of the length of ISe shortening was significantly higher in patients with a short length of ISe at baseline.

The length of ISe is a sensitive prognostic factor for the rate of ISe shortening in RP patients with EYS mutations. However, age, logMAR visual acuity, and MD value were not statistically significant prognostic factors in the present study, although a previous study has reported that the extent of visual fields constriction seemed to correlate better with age than with visual acuity. ${ }^{10}$ These findings help to explain the disease course to patients in clinical practice; that is, disease progression of RP patients with EYS mutations with relatively longer ISe should be slow, whereas that of the patients with relatively shorter ISe should be rapid. However, we cannot predict the degree of disease progression from age, visual acuity, and visual field. To expand the application of this approach, further studies in degenerative retinal diseases with other mutations are needed.

In this study, the rate of ISe shortening varied among patients with the same type of gene mutation, indicating that RP with the same gene mutation may not follow the same course in all patients. This finding is consistent with that in a previous study where patients with $P R P H 2$ mutations exhibited different phenotypes. ${ }^{19}$ The previous studies showed a wide range of phenotypic expression from the same mutation: central areolar choroidal dystrophy, autosomal dominant RP, adult vitelliform macular dystrophy, and cone-rod dystrophy.

Curcio et $a l^{20}$ showed that cone density decreased steeply with increasing eccentricity, whereas rod density increased with increasing eccentricity, and cone and rod density became the same at $0.5 \mathrm{~mm}$ from the fovea according to their published figure. Thus, an ISe shorter than $1000 \mu \mathrm{m}$ was mainly constituted of cone cells. The results of the present study suggested that cone cells were more easily disordered than rod cells. There is a need for longitudinal study of the decrease in photoreceptors in RP patients using an adaptive optics scanning laser ophthalmoscope.

In the present study, we selected the right eye in all cases to prevent selection bias. However, the symmetricity of progression of RP is also of interest. The ISe of left eye at baseline was $3180 \pm 2348 \mu \mathrm{m}$, which was not significantly different from that of the right eye (3137 $\pm 2348 \mu \mathrm{m}, P=0.67)$. The ISe of the left eye at final measurement was $2558 \pm 2325 \mu \mathrm{m}$, which was also not significantly different from that of the right eye
$(2456 \pm 2292 \mu \mathrm{m}, P=0.20)$. Thus, progression of RP in patients with EYS mutations was symmetric in the present study.

It is necessary to follow RP patients for at least 4 years to determine the true extent of changes, because RP is a disease with a long course. Indeed, because RP shows a definite but slow decline in visual function and degeneration of retinal structure, large clinical trials have set study periods for longer than 4 years, although their outcomes were different from those of this study. ${ }^{21-23}$

We converted the length of ISe to rate (\%) to exclude the effect of the length of ISe, because this study included patients with various stages of the disease. For instance, although a change in the length of ISe from 8000 to $7000 \mu \mathrm{m}$ indicates the same extent of progression as a reduction from 1500 to $500 \mu \mathrm{m}$, the implications are quite different. In fact, ISe at baseline was ranged from 481 to $7589 \mu \mathrm{m}$.

This study had some limitations. First, measurement of the length of ISe was manual. Because ISe may not have a clear-cut edge, the measurement of ISe length can incur errors. We attempted to minimize the level of error as much as possible by two means. One was to make use of the slope of the regression line derived from OCT images obtained at five time points over 5 years; analysis of only two data points, that is, baseline and $\sim 5$ years, would generate misleading results. The other was that the measurements of the length of ISe showed high repeatability; the $\operatorname{ICC}(2,1)$ value was 0.999 , indicating that almost the same length of ISe could be measured by any examiner. The measurement of the length of ISe is thus a relatively simple and facile, but accurate, method. Second, there were few young patients in this study. Although there was no significant correlation between the rate of ISe shortening and age at baseline, the rate of ISe shortening could not be determined in 7 of the 19 patients (37\%) in the EYS-RP group because of the absence of assessable ISe, whereas it could be detected when they were younger. If the results of these patients were included in their youth, the results of this study may be different. Suto et al ${ }^{10}$ reported that ISe was absent in $40 \%$ RP patients with EYS mutations. The results of the present study (37\%) were consistent with those of the previous report. Third, few patients had an intermediate length ISe at baseline. Among $12 \mathrm{RP}$ patients, 9 had ISe $<4000 \mu \mathrm{m}$, two had ISe $>6000 \mu \mathrm{m}$, and one had ISe ranged $4000-6000 \mu \mathrm{m}$. Further research of RP patients with middle ISe should be performed.

In conclusion, ISe of all RP patients with EYS mutations shortened during the 5 years of observation annually. The measurement of the length of ISe is a simple and convenient method with high repeatability, and this length is a sensitive prognostic factor for the rate of ISe shortening in RP patients with EYS mutations. 


\section{Summary}

What was known before

- The ISe band is correlated with visual function. EYS is an important and common cause of RP.

\section{What this study adds}

- The ISe band of RP patients with EYS mutations shortened during the 5 years of observation annually. The length of the ISe band is a sensitive prognostic factor for the rate of ISe shortening in RP patients with EYS mutations.

\section{Conflict of interest}

The authors declare no conflict of interest.

\section{Acknowledgements}

This work was partly supported by the Innovative Techno-Hub for Integrated Medical Bio-Imaging of the Project for Developing Innovation Systems, from the Ministry of Education, Culture, Sports, Science and Technology (MEXT), Japan. NY was financially supported by Topcon Corporation, Tokyo, Japan; Nidek, Gamagori, Japan (Consultant); and Canon, Tokyo, Japan.

\section{References}

1 Hartong DT, Berson EL, Dryja TP. Retinitis pigmentosa. Lancet 2006; 368: 1795-1809.

2 Kelsell RE, Gregory-Evans K, Payne AM, Perrault I, Kaplan J, Yang RB et al. Mutations in the retinal guanylate cyclase (RETGC-1) gene in dominant cone-rod dystrophy. Hum Mol Genet 1998; 7: 1179-1184.

3 Hayward C, Shu X, Cideciyan AV, Lennon A, Barran P, Zareparsi $\mathrm{S}$ et al. Mutation in a short-chain collagen gene, CTRP5, results in extracellular deposit formation in lateonset retinal degeneration: a genetic model for age-related macular degeneration. Hum Mol Genet 2003; 12: 2657-2667.

4 Xu Y, Guan L, Shen T, Zhang J, Xiao X, Jiang $\mathrm{H}$ et al. Mutations of 60 known causative genes in 157 families with retinitis pigmentosa based on exome sequencing. Hum Genet 2014; 133: 1255-1271.

5 Oishi M, Oishi A, Gotoh N, Ogino K, Higasa K, lida K et al. Comprehensive molecular diagnosis of a large cohort of Japanese retinitis pigmentosa and Usher syndrome patients by next-generation sequencing. Invest Ophthalmol Vis Sci 2014; 55: 7369-7375.

6 Arai Y, Maeda A, Hirami Y, Ishigami C, Kosugi S, Mandai M et al. Retinitis pigmentosa with EYS mutations is the most prevalent inherited retinal dystrophy in Japanese populations. J Ophthalmol 2015; 2015: 819760.

7 Barragán I, Borrego S, Pieras JI, González-del Pozo M, Santoyo J, Ayuso C et al. Mutation spectrum of EYS in Spanish patients with autosomal recessive retinitis pigmentosa. Hum Mutat 2010; 31: E1772-E1800.

8 Abd El-Aziz MM, O'Driscoll CA, Kaye RS, Barragan I, ElAshry MF, Borrego $S$ et al. Identification of novel mutations in the ortholog of drosophila eyes shut gene (EYS) causing autosomal recessive retinitis pigmentosa. Invest Ophthalmol Vis Sci 2010; 51: 4266-4272.

9 Bandah-Rozenfeld D, Littink KW, Ben-Yosef T, Strom TM, Chowers I, Collin RW et al. Novel null mutations in the EYS gene are a frequent cause of autosomal recessive retinitis pigmentosa in the Israeli population. Invest Ophthalmol Vis Sci 2010; 51: 4387-4394.

10 Suto K, Hosono K, Takahashi M, Hirami Y, Arai Y, Nagase Y et al. Clinical phenotype in ten unrelated Japanese patients with mutations in the EYS gene. Ophthalmic Genet 2014; 35: 25-34.

11 Ogino K, Oishi A, Oishi M, Gotoh N, Morooka S, Sugahara $\mathrm{M}$ et al. Efficacy of column scatter plots for presenting retinitis pigmentosa phenotypes in a Japanese cohort. Transl Vis Sci Technol 2016; 5: 4. eCollection 2016.

12 Birch DG, Locke KG, Wen Y, Locke KI, Hoffman DR, Hood DC. Spectral-domain optical coherence tomography measures of outer segment layer progression in patients with X-linked retinitis pigmentosa. JAMA Ophthalmol 2013; 131: $1143-1150$.

13 Birch DG, Locke KG, Felius J, Klein M, Wheaton DK, Hoffman DR et al. Rates of decline in regions of the visual field defined by frequency-domain optical coherence tomography in patients with RPGR-mediated X-linked retinitis pigmentosa. Ophthalmology 2015; 122: 833-839.

14 Sandberg MA, Brodkhurst RJ, Gaudio AR, Berson EL. The association between visual acuity and central retinal thickness in retinitis pigmentosa. Invest Ophthalmol Vis Sci 2005; 46: 3349-3354.

15 Murakami T, Akimoto M, Ooto S, Suzuki T, Ikeda H, Kawagoe $\mathrm{N}$ et al. Association between abnormal autofluorescence and photoreceptor disorganization in retinitis pigmentosa. Am J Ophthalmol 2008; 145: 687-694.

16 Aizawa S, Miytamura Y, Baba T, Hagiwara A, Ogata K, Yamamoto S. Correlation between visual function and photoreceptor inner/outer segment junction in patients with retinitis pigmentosa. Eye 2009; 23: 304-308.

17 Rangaswamy NV, Patel HM, Locke KG, Hood DC, Birch DG. A comparison of visual field sensitivity to photoreceptor thickness in retinitis pigmentosa. Invest Ophthalmol Vis Sci 2010; 51: 4213-4219.

18 Hood DC, Ramachandran R, Holopigian K, Lazow M, Birch DG, Greenstein VC. Method for deriving visual field boundaries from OCT scans of patients with retinitis pigmentosa. Biomed Opt Express 2011; 2: 1106-1114.

19 Renner AB, Fiebig BS, Weber BH, Wissinger B, Andreasson $\mathrm{S}$, Gal A et al. Phenotypic variability and long-term followup of patients with known and novel PRPH2/RDS gene mutations. Am J Ophthalmol 2009; 147: 518-530.

20 Curcio CA, Sloan KR, Kalina RE, Hendrickson AE. Human photoreceptor topography. J Comp Neurol 1990; 292: 497-523.

21 Berson EL, Rosner B, Sandberg MA, Hayes KC, Nicholson BW, Weigel-DiFranco $C$ et al. A randomized trial of vitamin $\mathrm{A}$ and vitamin $\mathrm{E}$ supplementation for retinitis pigmentosa. Arch Ophthalmol 1993; 111: 761-772.

22 Berson EL, Rosner B, Sandberg MA, Weigel-DiFranco C, Moser A, Brockhurst RJ et al. Clinical trial of docosahexaenoic acid in patients with retinitis pigmentosa receiving vitamin A treatment. Arch Ophthalmol 2004; 122: 1297-1305.

23 Berson EL, Rosner B, Sandberg MA, Weigel-DiFranco C, Brockhurst RJ, Hayes KC et al. Clinical trial of lutein in patients with retinitis pigmentosa receiving vitamin A. Arch Ophthalmol 2010; 128: 403-411. 